\title{
A METHOD OF IMAGES FOR THE EVALUATION OF ELECTROSTATIC FIELDS IN SYSTEMS OF CLOSELY SPACED CONDUCTING CYLINDERS*
}

\author{
HONGWEI CHENG ${ }^{\dagger}$ AND LESLIE GREENGARD ${ }^{\ddagger}$
}

\begin{abstract}
A long-standing area of materials science research has been the study of electrostatic, magnetic, and elastic fields in composites with densely packed inclusions whose material properties differ markedly from that of the background. While powerful tools exist for dilute suspensions, accurate calculations in the close-to-touching case have been carried out largely by asymptotic methods and only for simple geometries such as regular arrays of cylinders or spheres. In this paper, we develop a hybrid numerical method for the evaluation of electrostatic fields in composites consisting of arbitrary dispersions of cylinders. Our approach is based on an integral equation formulation of the governing interface problem combined with a new method of images. High accuracy is achieved using only a small number of degrees of freedom for each inclusion.
\end{abstract}

Key words. method of images, electrostatics, composite materials

AMS subject classifications. 31A10, 35J05, 65R20, 65P05

PII. S0036139996297614

1. Introduction. As materials with complex microstructures become easier and easier to manufacture, there is a corresponding need for analytic and numerical methods that allow us to predict and understand their properties. In this paper, we will concentrate on the determination of electrical transport properties in a specific twodimensional setting, namely a composite consisting of a uniform background in which are embedded cylindrical inclusions with distinct conductivities which may be very close to touching. We leave the translation of our results to other applications (such as heat transport) to the reader $[1,9,17]$.

To fix notation, consider a composite material consisting of a uniform background matrix with conductivity $\sigma_{e}$ in which are embedded $M$ cylindrical inclusions $D_{i}$ centered at the points $\left\{\left(x_{i}, y_{i}\right)\right\}$ and having conductivities $\sigma_{i}$ for $i=1, \ldots, M$. We will assume, for the sake of simplicity, that the radii of the cylinders (disks) are identically equal to $a$, but it is straightforward to generalize the method below to the case where the cylinders are not equisized. The electric field induced in such a material by a uniform field applied in the $x$-direction can be obtained by solving the interface problem:

$$
\begin{aligned}
& \Delta u_{e}=0 \quad \text { in } \quad \mathbf{R}^{2}-\bigcup_{j=1}^{M} D_{j}, \\
& \Delta u_{j}=0 \quad \text { in } \quad D_{j}, \quad j=1,2, \ldots, M,
\end{aligned}
$$

* Received by the editors January 26, 1996; accepted for publication (in revised form) August 16, 1996. This research was supported by the Applied Mathematical Sciences Program of the U.S. Department of Energy under contract DEFGO288ER25053.

http://www.siam.org/journals/siap/57-6/29761.html

$\dagger$ Courant Institute of Mathematical Sciences, New York University, New York, NY 10012. Present address: Department of Civil Engineering and Operations Research, Princeton University, Princeton, NJ 08544 (hongwei@cherrypit.princeton.edu).

${ }^{\ddagger}$ Courant Institute of Mathematical Sciences, New York University, New York, NY 10012 (greengard@cims.nyu.edu). The research of this author was supported in part by an NSF Presidential Young Investigator Award and a Packard Foundation Fellowship. 


$$
\begin{aligned}
u_{e} & =u_{j} \quad \text { on } \quad \partial D_{j}, \quad j=1,2, \ldots, M \\
\sigma_{e} \frac{\partial u_{e}}{\partial n} & =\sigma_{j} \frac{\partial u_{j}}{\partial n} \quad \text { on } \quad \partial D_{j}, \quad j=1,2, \ldots, M
\end{aligned}
$$

with the far field condition

$$
\nabla u_{e}(P) \longrightarrow(1,0) \text { as } P \longrightarrow \infty
$$

where $u_{j}$ denotes the potential restricted to the inclusion $D_{j}, u_{e}$ denotes the potential restricted to the matrix, and $\partial / \partial n$ denotes the outward normal derivative on the boundary of the inclusion. The global potential function will be referred to as $u$.

Classical potential theory $[11,12,35]$ suggests representing the solution to the system (1)-(5) in terms of a single layer potential

$$
u(P)=x+\sum_{j=1}^{M} \int_{\partial D_{j}} G(P, Q) \rho_{j}(Q) d s,
$$

where $G(P, Q)=\frac{1}{2 \pi} \log |P-Q|$ is the free-space Green function and $\rho$ is the charge density which remains to be determined. Equations (1)-(3) are automatically satisfied, and imposition of the remaining interface condition yields the system of integral equations

$$
\begin{array}{cc}
2 \lambda_{1} n_{1}(P) & =\rho_{1}(P)-2 \lambda_{1} \sum_{j=1}^{M} \int_{\partial D_{j}} \frac{\partial G}{\partial n}(P, Q) \rho_{j}(Q) d s, \quad P \in \partial D_{1}, \\
\ldots & \\
2 \lambda_{M} n_{1}(P) & =\rho_{M}(P)-2 \lambda_{M} \sum_{j=1}^{M} \int_{\partial D_{j}} \frac{\partial G}{\partial n}(P, Q) \rho_{j}(Q) d s, \quad P \in \partial D_{M},
\end{array}
$$

where $\lambda_{j}=\frac{\sigma_{j}-\sigma_{e}}{\sigma_{j}+\sigma_{e}}$, and $n_{1}(P)$ denotes the $x$-component of the unit outward normal at $P$.

The representation (6) can, of course, be used for inclusions of arbitrary shape. In the multidisk problem, however, we can expand the charge density on the $j$ th inclusion as a Fourier series which we express in complex notation as

$$
\rho_{j}(Q)=\operatorname{Re}\left(\sum_{k=1}^{\infty} A_{j}(k) e^{i k \theta}\right) .
$$

Here, the multipole moments (or Fourier coefficients) $A_{j}(k)$ are unknown complex numbers and $Q=\left(x_{j}+a \cos \theta, y_{j}+a \sin \theta\right)$. No constant term is needed in the Fourier series, since $\rho$ can be shown to be charge neutral. Substituting the representation (8) into the integral equation (7) and some analytic manipulation yield the infinitedimensional linear system

$$
A_{j}(k)+\lambda_{j} \sum_{\substack{m=1 \\
m \neq j}}^{M} \sum_{l=1}^{\infty}\left(\begin{array}{c}
l+k-1 \\
k-1
\end{array}\right)\left(\frac{a}{\mathbf{z}_{m}-\mathbf{z}_{j}}\right)^{l+k}(-1)^{k} \overline{A_{m}(l)}= \begin{cases}2 \lambda_{j} & \text { if } k=1, \\
0 & \text { if } k>1,\end{cases}
$$

where $\mathbf{z}_{j}=x_{j}+i y_{j}$ is the center of $D_{j}$ viewed as a point in the complex plane. This method, which we will loosely refer to as Rayleigh's method, is capable of describing the electrostatic field for any configuration of disks and any prescribed conductivities. 
Remark 1.1. In the case of a single disk, the system (9) has the analytic solution

$$
A_{1}(1)=2 \lambda_{1}, \quad A_{1}(k)=0 \text { for } k>1,
$$

or

$$
\rho_{1}(Q)=2 \lambda_{1} \cos \theta .
$$

In other words, the polarization charge induced on the surface of the inclusion by the applied field is that of a pure dipole.

Remark 1.2. It should be noted that Lord Rayleigh, in his classic paper [28], introduced a further modification of this Fourier approach in order to determine the effective conductivity of a composite material consisting of a periodic array of disks in a uniform background. Applications of Rayleigh's method to more complex periodic systems can be found in $[19,21,30,32]$. While we will consider periodic geometries in section 3.2, we will restrict our attention, for the moment, to the infinite medium problem.

In practice, it is clear that the infinite system (9) has to be made finite by ignoring all multipole moments beyond a given order. In the case that the inclusions are reasonably well separated or have conductivities close to that of the background, the number of moments which need to be retained to resolve the charge density is relatively small, and Rayleigh's method gives excellent results. Unfortunately, if the inclusions are close to touching and their conductivities differ greatly from that of the background, the charge density becomes nearly singular and the number of degrees of freedom required grows extremely large. The linear system to be solved also becomes ill conditioned. If we were to consider a system of 100 inclusions with unit radius, each within a distance $10^{-6}$ of at least one other, we would need millions of Fourier modes to obtain reliable results. This is clearly unsatisfactory.

Most of the methods which have been developed to circumvent this difficulty are based on asymptotics $[2,4,13,14,20,23,24,31]$ that correctly describe the closeto-touching interaction for special geometries. However, there has been no efficient method that allows for accurate evaluation of the field in arbitrary dispersions. Even fast multipole-accelerated integral equation methods [7], whose cost grows linearly with the number of unknowns, can only make limited progress here because the number of degrees of freedom grows so rapidly.

In the remainder of this paper, we present a new numerical method for the evaluation of electrostatic fields in densely packed dispersions, which overcomes the difficulties associated with Rayleigh's method. The mathematical foundations of our approach are presented in section 2, where we extend the classical method of images for dipole sources to multipoles of arbitrary order. In section 3, we describe our hybrid integral equation scheme, and in section 4 we present some numerical examples for both clusters in an infinite medium and periodic composites. Section 5 contains some concluding remarks.

2. The method of images. A different approach to solving the multidisk interface problem (1)-(5) begins with the single disk solution given by (10). In the infinitely dilute limit, the multidisk solution is well approximated by the superposition of single disk solutions, namely,

$$
\rho_{j} \approx 2 \lambda_{j} \cos \theta_{j},
$$

where $\left(r_{j}, \theta_{j}\right)$ are the coordinates of the point $(r, \theta)$ with respect to the $j$ th disk center. This gives rise to an approximation of the global potential function of the form 

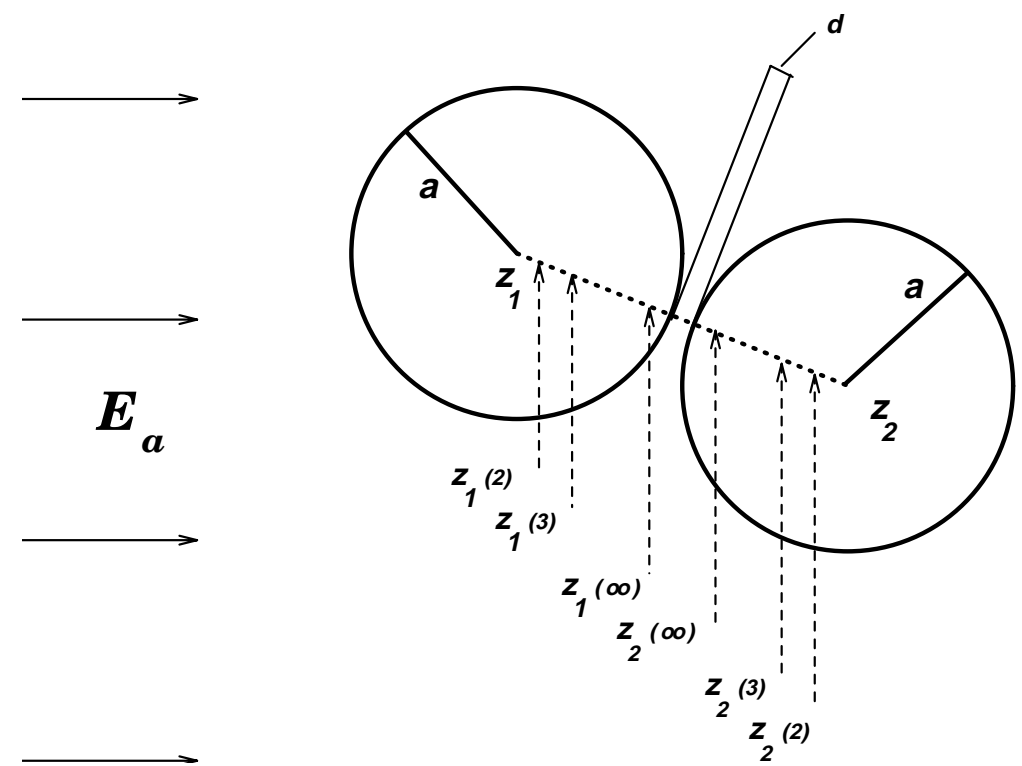

Fig. 1. Two disks separated by a distance $d$ and the image series generated by successive reflection.

$$
\begin{aligned}
& u_{e}(r, \theta) \approx r \cos \theta+\sum_{j=1}^{M} a^{2} \lambda_{j} \frac{\cos \theta_{j}}{r_{j}}, \\
& u_{j}(r, \theta) \approx r \cos \theta+\lambda_{j} r_{j} \cos \theta_{j} .
\end{aligned}
$$

Thus, each dipole distribution of surface charge of the form (11) yields a point dipole approximation of the external field (12).

The infinitely dilute approximation must be abandoned, however, as the inclusions approach each other and begin to interact more and more strongly. Rather than include higher-order multipole corrections as in Rayleigh's method, the method of images $[10,18,22,24]$ proceeds by representing the field in terms of a series of dipole fields. For ease of notation, we equate the plane $\mathbf{R}^{2}$ with the complex plane $\mathbf{C}$.

LEMMA 2.1. Let $D_{1}$ and $D_{2}$ be nonintersecting disks of radius a centered at $\mathbf{z}_{1}$ and $\mathbf{z}_{2}$ with identical conductivities $\sigma_{d}$ (Fig. 1). Then, in the presence of a uniform applied field $\mathbf{E}_{a}=(1,0)$, the potential is given by

$$
\begin{aligned}
& u_{e}=\operatorname{Re}\left(\mathbf{z}+\sum_{k=1}^{\infty} \frac{\alpha_{1}^{(k)}}{\mathbf{z}-\mathbf{z}_{1}(k)}+\sum_{k=1}^{\infty} \frac{\alpha_{2}^{(k)}}{\mathbf{z}-\mathbf{z}_{2}(k)}\right), \\
& u_{1}=\operatorname{Re}\left(\mathbf{z}+\lambda\left(\mathbf{z}-\mathbf{z}_{1}\right)+(1-\lambda) \sum_{k=1}^{\infty} \frac{\alpha_{2}^{(k)}}{\mathbf{z}-\mathbf{z}_{2}(k)}\right), \\
& u_{2}=\operatorname{Re}\left(\mathbf{z}+\lambda\left(\mathbf{z}-\mathbf{z}_{2}\right)+(1-\lambda) \sum_{k=1}^{\infty} \frac{\alpha_{1}^{(k)}}{\mathbf{z}-\mathbf{z}_{1}(k)}\right),
\end{aligned}
$$

where

$$
\alpha_{1}^{(1)}=a^{2} \lambda, \quad a_{2}^{(1)}=a^{2} \lambda, \quad \mathbf{z}_{1}(1)=\mathbf{z}_{1}, \quad \mathbf{z}_{2}(1)=\mathbf{z}_{2},
$$


and, for $k>1$,

$$
\begin{aligned}
\mathbf{z}_{1}(k) & =\mathbf{z}_{1}+a^{2} /\left(\overline{\mathbf{z}_{2}(k-1)-\mathbf{z}_{1}}\right), \\
\mathbf{z}_{2}(k) & =\mathbf{z}_{2}+a^{2} /\left(\overline{\mathbf{z}_{1}(k-1)-\mathbf{z}_{2}}\right), \\
\alpha_{1}^{(k)} & =\lambda a^{2} \cdot \overline{\alpha_{2}^{(k-1)} /\left(\mathbf{z}_{1}(k-1)-\mathbf{z}_{2}\right)^{2}}, \\
\alpha_{2}^{(k)} & =\lambda a^{2} \cdot \overline{\alpha_{1}^{(k-1)} /\left(\mathbf{z}_{2}(k-1)-\mathbf{z}_{1}\right)^{2}} .
\end{aligned}
$$

The limiting image points are given by

$$
\begin{aligned}
& \mathbf{z}_{1}(\infty)=\frac{\mathbf{z}_{1}+\mathbf{z}_{2}}{2}-\sqrt{a d+d^{2} / 4} \cdot \frac{\mathbf{z}_{2}-\mathbf{z}_{1}}{\left|\mathbf{z}_{2}-\mathbf{z}_{1}\right|}, \\
& \mathbf{z}_{2}(\infty)=\frac{\mathbf{z}_{1}+\mathbf{z}_{2}}{2}+\sqrt{a d+d^{2} / 4} \cdot \frac{\mathbf{z}_{2}-\mathbf{z}_{1}}{\left|\mathbf{z}_{2}-\mathbf{z}_{1}\right|},
\end{aligned}
$$

where $d=\left|\mathbf{z}_{2}-\mathbf{z}_{1}\right|-2 a$ is the distance between the two disks.

Proof. Ignoring all terms with $k \geq 2$ in (14) corresponds to the dilute approximation of (12) and (13), which fails to satisfy the interface conditions (3) and (4) on either disk. The method of images proceeds by reflecting the dipole source at $\mathbf{z}_{2}$ into the point $\mathbf{z}_{1}(2)$ in $D_{1}$ with strength $a_{1}(2)$. A straightforward calculation shows that the functions

$$
\begin{aligned}
& u_{e}^{(1)}=\operatorname{Re}\left(\mathbf{z}+\sum_{k=1}^{2} \frac{\alpha_{1}^{(k)}}{\mathbf{z}-\mathbf{z}_{1}(k)}+\sum_{k=1}^{1} \frac{\alpha_{2}^{(k)}}{\mathbf{z}-\mathbf{z}_{2}(k)}\right), \\
& u_{1}^{(1)}=\operatorname{Re}\left(\mathbf{z}+\lambda\left(\mathbf{z}-\mathbf{z}_{1}\right)+(1-\lambda) \sum_{k=1}^{1} \frac{\alpha_{2}^{(k)}}{\mathbf{z}-\mathbf{z}_{2}(k)}\right), \\
& u_{2}^{(1)}=\operatorname{Re}\left(\mathbf{z}+\lambda\left(\mathbf{z}-\mathbf{z}_{2}\right)\right)
\end{aligned}
$$

satisfy the continuity conditions on $D_{1}$ but not on $D_{2}$. Reflection of the original dipole source at $\mathbf{z}_{1}$ plus the image at $\mathbf{z}_{1}(2)$ into $D_{2}$ yields the functions

$$
\begin{aligned}
& u_{e}^{(2)}=\operatorname{Re}\left(\mathbf{z}+\sum_{k=1}^{2} \frac{\alpha_{1}^{(k)}}{\mathbf{z}-\mathbf{z}_{1}(k)}+\sum_{k=1}^{3} \frac{\alpha_{2}^{(k)}}{\mathbf{z}-\mathbf{z}_{2}(k)}\right), \\
& u_{1}^{(2)}=\operatorname{Re}\left(\mathbf{z}+\lambda\left(\mathbf{z}-\mathbf{z}_{1}\right)+(1-\lambda) \sum_{k=1}^{1} \frac{\alpha_{2}^{(k)}}{\mathbf{z}-\mathbf{z}_{2}(k)}\right), \\
& u_{2}^{(2)}=\operatorname{Re}\left(\mathbf{z}+\lambda\left(\mathbf{z}-\mathbf{z}_{2}\right)+(1-\lambda) \sum_{k=1}^{2} \frac{\alpha_{1}^{(k)}}{\mathbf{z}-\mathbf{z}_{1}(k)}\right),
\end{aligned}
$$

which now satisfy the flux conditions on $D_{2}$ but not on $D_{1}$. Continuation of this process leads to the solution (14). (These series are easily seen to converge, since the magnitudes of successive terms are decaying geometrically.) The formulae for $\mathbf{z}_{1}(\infty)$ and $\mathbf{z}_{2}(\infty)$ are obtained by finding a fixed point for the reflection procedure.

Let us establish the nature of the relationship between Rayleigh's method and the method of images for the two-disk problem. The former is based on seeking the global potential $u$ in the form of a single layer potential

$$
u(P)=x+\sum_{j=1}^{2} \int_{\partial D_{j}} G(P, Q) \rho_{j}(Q) d s,
$$


where $G(P, Q)$ is the free-space Green function and $\rho$ is the charge density. While the kernel $G(P, Q)$ is simple to evaluate, $\rho$ becomes very complex in the close-to-touching case, requiring many Fourier modes to resolve. The method of images also comes from an integral representation

$$
u(P)=x+\sum_{j=1}^{2} \int_{\partial D_{j}} K(P, Q) \mu_{j}(Q) d s,
$$

but $K(P, Q)$ can be thought of as incorporating the influence of all images according to (14). Inserting the integral representation (19) into the system (7), with $\partial G / \partial n$ replaced by $\partial K / \partial n$, yields the analytic solution

$$
\mu_{j}(Q)=2 \lambda_{j} \cos \theta,
$$

where $Q=\left(x_{j}+a \cos \theta, y_{j}+a \sin \theta\right)$. By sacrificing simplicity of the kernel, we are able to invert the integral equation analytically and to expand $\mu_{i}$ with only one Fourier mode. In the language of functional analysis, $K(P, Q)$ is the resolvent kernel for the integral equation (7).

Unfortunately, the multidisk case is not so simple. To see this, suppose that we compute the image series generated by each pair of disks using Lemma 2.1, which we refer to as first-order reflections. These images must then themselves be reflected into all other disks, generating second-order reflections, and so on. Even if we truncated each image series after a finite number of terms, the total number of reflections required grows exponentially with the number of disks. Thus, the method of images is impractical for large-scale problems.

2.1. A reflection theorem. Rayleigh's method and the method of images are clearly at opposite extremes of the spectrum of possible integral representations. It would seem reasonable, therefore, to investigate the behavior of a method which is situated somewhere in between, such as one where images are used for each pair of close-to-touching disks but not for any other interactions. This has an obvious interpretation in terms of the kernel of the integral equation (7). As an example, consider the three-disk problem of Fig. 2.

Because of its spatial separation from the other disks, the surface charge density on disk $D_{3}$ is well represented by only a small number of Fourier modes. The interaction between disks $D_{1}$ and $D_{2}$, on the other hand, is more complex and better handled by an image series (indicated in Fig. 2 by a dotted line). Therefore, we choose a global integral representation of the form

$$
u(P)=x+\sum_{j=1}^{3} \int_{\partial D_{j}} K_{j}(P, Q) \mu_{j}(Q) d s,
$$

where $K_{3}(P, Q)$ is just $\frac{1}{2 \pi} \log |P-Q|$, but $K_{1}(P, Q)$ and $K_{2}(P, Q)$ include the influence of all images. Note that, in order to be a self-consistent representation, the influence of the images placed in disks $D_{1}$ and $D_{2}$ must be considered not only on each other, but on $D_{3}$ as well.

There is, however, an analytical obstacle to using this idea: the densities $\mu_{j}$ will no longer be pure dipole distributions and the formulae of Lemma 2.1 do not apply. On the other hand, each $\mu_{j}$ is still a charge distribution on $\partial D_{j}$ whose far field can be expressed as a multipole expansion. The following theorem, which does not seem to be 

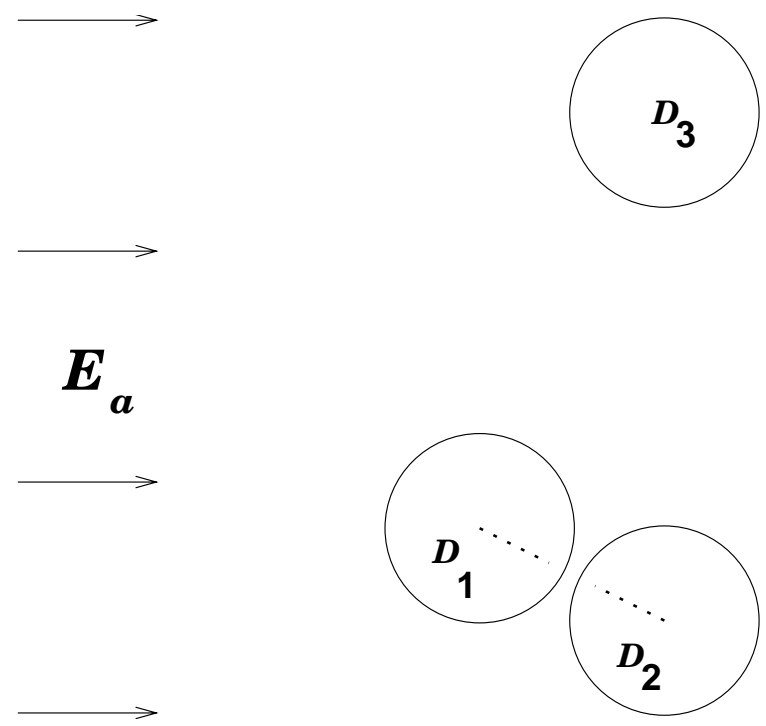

FIG. 2. Three disks in a uniform applied field $\mathbf{E}_{a}=(1,0)$.

available in the literature, gives a simple formula for reflecting a multipole expansion of arbitrary order.

THEOREM 2.2. Suppose that $D_{1}$ is a disk of radius a, centered at $\mathbf{z}_{1}$, with conductivity $\sigma_{1}$, embedded in the infinite medium $\mathbf{C}$ of conductivity $\sigma_{e}$. Suppose also that $\lambda=\left(\sigma_{1}-\sigma_{e}\right) /\left(\sigma_{1}+\sigma_{e}\right)$ and that $\Phi$ is a multipole source centered at $\mathbf{z}_{2}$ with $\left|\mathbf{z}_{1}-\mathbf{z}_{2}\right|>a$,

$$
\Phi(\mathbf{z})=\sum_{k=1}^{p} \frac{\alpha(k)}{\left(\mathbf{z}-\mathbf{z}_{2}\right)^{k}} .
$$

Let $\mathbf{z}_{I}=\mathbf{z}_{1}+a^{2} /\left(\overline{\mathbf{z}_{2}}-\overline{\mathbf{z}_{1}}\right)$ and let

$$
\Phi_{I}(\mathbf{z})=\sum_{k=0}^{p} \frac{\beta(k)}{\left(\mathbf{z}-\mathbf{z}_{I}\right)^{k}},
$$

where $\beta(k)$ satisfies

$$
\overline{\beta(k)}=-\lambda\left(\frac{a^{2}}{\mathbf{z}_{2}-\mathbf{z}_{1}}\right)^{k} \sum_{m=k}^{p}\left(\begin{array}{c}
m \\
k
\end{array}\right)\left(\frac{-1}{\mathbf{z}_{2}-\mathbf{z}_{1}}\right)^{m} \alpha(m)
$$

for $k=0,1, \ldots, p$. Then the functions

$$
\begin{aligned}
& u_{e}=\operatorname{Re}\left(\Phi(\mathbf{z})+\Phi_{I}(\mathbf{z})\right), \\
& u_{1}=\operatorname{Re}((1-\lambda) \Phi(\mathbf{z}))
\end{aligned}
$$

are harmonic in $\mathbf{C}-\left(D_{1} \cup \mathbf{z}_{2}\right)$ and $D_{1}$, respectively, and satisfy the interface conditions

$$
\begin{aligned}
& u_{e}=u_{1} \quad \text { on } \partial D_{1}, \\
& \sigma_{e} \frac{\partial u_{e}}{\partial n}=\sigma_{1} \frac{\partial u_{1}}{\partial n} \quad \text { on } \quad \partial D_{1} \text {. }
\end{aligned}
$$


Proof. For the sake of simplicity, we first translate $\mathbf{z}_{1}$ to the origin. The circle theorem [25] (or the method of inversion [18]) then states that if $\Phi(\mathbf{z})$ is an analytic function inside $D_{1}$, then the desired interface conditions are satisfied by

$$
\begin{aligned}
& u_{e}=\operatorname{Re}\left(\Phi(\mathbf{z})-\lambda \overline{\Phi\left(a^{2} / \overline{\mathbf{z}}\right)}\right), \\
& u_{1}=\operatorname{Re}((1-\lambda) \Phi(\mathbf{z})) .
\end{aligned}
$$

Thus,

$$
\Phi_{I}(\mathbf{z})=-\lambda \overline{\sum_{k=0}^{p} \frac{\alpha(k)}{\left(\frac{a^{2}}{\overline{\mathbf{z}}}-\mathbf{z}_{2}\right)^{k}}} .
$$

A sequence of algebraic manipulations yields

$$
\begin{aligned}
\Phi_{I}(\mathbf{z}) & =-\lambda \sum_{k=0}^{p} \frac{\bar{\alpha}(k)}{\left(\frac{a^{2}}{\mathbf{z}}-\overline{\mathbf{z}}_{2}\right)^{k}} \\
& =-\lambda \sum_{k=0}^{p} \frac{\bar{\alpha}(k)}{\left(\frac{a^{2}}{\overline{\mathbf{z}}_{2}}-\mathbf{z}\right)^{k}\left(\frac{\left.\overline{\mathbf{z}}_{2}\right)^{k}}{\mathbf{z}}\right.} \\
& =-\lambda \sum_{k=0}^{p} \frac{\bar{\alpha}(k)}{\left(-\overline{\mathbf{z}}_{2}\right)^{k}} \frac{\mathbf{z}^{k}}{\left(\mathbf{z}-\mathbf{z}_{I}\right)^{k}} \\
& =-\lambda \sum_{k=0}^{p} \frac{\bar{\alpha}(k)}{\left(-\overline{\mathbf{z}}_{2}\right)^{k}} \frac{\sum_{m=0}^{k}\left(\begin{array}{c}
k \\
m
\end{array}\right) \mathbf{z}_{I}^{m}\left(\mathbf{z}-\mathbf{z}_{I}\right)^{k-m}}{\left(\mathbf{z}-\mathbf{z}_{I}\right)^{k}} \\
& =-\lambda \sum_{k=0}^{p} \sum_{m=0}^{k}\left(\begin{array}{c}
k \\
m
\end{array}\right) \frac{\bar{\alpha}(k)}{\left(-\overline{\mathbf{z}}_{2}\right)^{k}} \frac{\mathbf{z}_{I}^{m}}{\left(\mathbf{z}-\mathbf{z}_{I}\right)^{m}} .
\end{aligned}
$$

Rearranging the order of the summation,

$$
\Phi_{I}(\mathbf{z})=-\lambda \sum_{k=0}^{p}\left[\left(\frac{a^{2}}{\overline{\mathbf{z}}_{2}}\right)^{k} \sum_{m=k}^{p}\left(\begin{array}{c}
m \\
k
\end{array}\right) \frac{\bar{\alpha}(m)}{\left(-\overline{\mathbf{z}}_{2}\right)^{m}}\right] \frac{1}{\left(\mathbf{z}-\mathbf{z}_{I}\right)^{k}} .
$$

Remark 2.1. Note that the preceding theorem is exact; a finite-order multipole has a finite-order image.

Remark 2.2. As noted earlier, the multipole expansion $\Phi(\mathbf{z})$ in the preceding theorem is generally derived from a charge distribution, say $\mu_{2}$, on the boundary of a nearby disk, say $\partial D_{2}$. An alternative derivation of formula (22) would be to compute the image charge distribution on the reflection of $D_{2}$ in $D_{1}$, which we refer to as the image disk. The image multipole series $\Phi_{I}(\mathbf{z})$ could then be obtained as the far field expansion induced by this image charge distribution. The center of the image disk is $\mathbf{z}_{1}(2)$ in (15), and it is easy to verify that the radius of the image disk on reflection is less than $a / 2$ (see Fig. 3).

DEFINITION 2.1. The operator that maps the mulitpole coefficients $\alpha=$ $(\alpha(1), \ldots, \alpha(p))$ to the coefficients $\beta=(\beta(1), \ldots, \beta(p))$ according to formula $(22)$ will be referred to as as the reflection operator $\mathcal{R}_{\mathbf{z}_{1}, \mathbf{z}_{2}}=\mathcal{R}\left(\mathbf{z}_{1}, \mathbf{z}_{2}, a\right)$.

The following lemma follows immediately from the preceding definition and Theorem 2.2. 
LEMmA 2.3. Let $D_{1}$ and $D_{2}$ be nonintersecting disks of radius a, centered at $\mathbf{z}_{1}$ and $\mathbf{z}_{2}$, with identical contrast parameter $\lambda$ (Fig. 1). Suppose further that there is a multipole source

$$
\Phi^{(1)}(\mathbf{z})=\sum_{k=1}^{p} \frac{\alpha^{(1)}(k)}{\left(\mathbf{z}-\mathbf{z}_{2}\right)^{k}}
$$

centered at $\mathbf{z}_{2}$. Then successive reflection in $D_{1}$ and $D_{2}$ generates a series of image multipole expansions given by

$$
\Phi^{(m)}(\mathbf{z})=\sum_{k=1}^{p} \frac{\alpha^{(m)}(k)}{\left(\mathbf{z}-\zeta_{m}\right)^{k}},
$$

where

$$
\zeta_{m}=\left\{\begin{array}{l}
\mathbf{z}_{2}(m) \quad \text { if } m \text { is odd, } \\
\mathbf{z}_{1}(m) \text { if } m \text { is even, }
\end{array}\right.
$$

$\mathbf{z}_{1}(m)$ and $\mathbf{z}_{2}(m)$ are defined in (15), and $\alpha^{(m)}=\left(\alpha^{(m)}(1), \ldots, \alpha^{(m)}(p)\right)$ is defined recursively by

$$
\alpha^{(m)}= \begin{cases}\mathcal{R}_{\mathbf{z}_{2}, \zeta_{m-1}} \alpha^{(m-1)} & \text { if } m \text { is odd } \\ \mathcal{R}_{\mathbf{z}_{1}, \zeta_{m-1}} \alpha^{(m-1)} & \text { if } m \text { is even }\end{cases}
$$

for $m \geq 2$.

3. A hybrid numerical method. We are now in a position to consider the approach suggested at the beginning of section 2.1 in a systematic fashion. Letting $\delta$ be a free parameter, we represent the potential for a multidisk problem as

$$
u(P)=x+\sum_{j=1}^{M} \int_{\partial D_{j}} K_{j}^{\delta}(P, Q) \mu_{j}(Q) d s,
$$

where $K_{j}^{\delta}(P, Q)$ includes the influence of all first-order reflections generated by interactions with disks which lie within a distance $\delta$ of $D_{j}$. In practice, we choose $\delta=a$, where $a$ is the disk radius. (In the absence of nearby disks, $K_{j}^{\delta}(P, Q)$ is just the free-space kernel.) More precisely, we expand $\mu_{j}$ as a finite Fourier series on $\partial D_{j}$,

$$
\mu_{j}(Q)=\operatorname{Re}\left(\sum_{k=1}^{p} \hat{\mu}_{j}(k) e^{i k \theta}\right),
$$

where $Q=\left(x_{j}+a \cos \theta, y_{j}+a \sin \theta\right)$. A straightforward calculation shows that the far field induced by the charge density $\mu_{j}$ on $\partial D_{j}$ is given by the multipole expansion of degree $p$

$$
\Phi(\mathbf{z})=\operatorname{Re}\left(\sum_{k=1}^{p} \frac{\alpha_{j}(k)}{\left(\mathbf{z}-\mathbf{z}_{j}\right)^{k}}\right),
$$

where

$$
\alpha_{j}(k)=-\frac{a^{k+1} \overline{\hat{\mu}_{j}(k)}}{2 k} .
$$


The first-order reflections in the integral representation for $u$ are an image series like (14) but where the reflections are obtained according to formula (23). For illustration, the three-disk problem depicted in Fig. 2 would be solved by letting

$$
\begin{aligned}
u_{e} & =\operatorname{Re}\left(\mathbf{z}+\sum_{m=1}^{N} \sum_{k=1}^{p} \frac{\alpha_{1}^{(m)}(k)}{\left(\mathbf{z}-\mathbf{z}_{1}(m)\right)^{k}}+\sum_{m=1}^{N} \sum_{k=1}^{p} \frac{\alpha_{2}^{(m)}(k)}{\left(\mathbf{z}-\mathbf{z}_{2}(m)\right)^{k}}+\sum_{k=1}^{p} \frac{\alpha_{3}(k)}{\left(\mathbf{z}-\mathbf{z}_{3}\right)^{k}}\right), \\
u_{1} & =\operatorname{Re}\left(\mathbf{z}+\sum_{k=1}^{p} \frac{\overline{\alpha_{1}(k)}}{a^{2 k}}\left(\mathbf{z}-\mathbf{z}_{1}\right)^{k}+(1-\lambda) \sum_{m=1}^{N} \sum_{k=1}^{p} \frac{\alpha_{2}^{(m)}(k)}{\left(\mathbf{z}-\mathbf{z}_{2}(m)\right)^{k}}+\sum_{k=1}^{p} \frac{\alpha_{3}(k)}{\left(\mathbf{z}-\mathbf{z}_{3}\right)^{k}}\right), \\
(26) & \\
u_{2} & =\operatorname{Re}\left(\mathbf{z}+\sum_{k=1}^{p} \frac{\overline{\alpha_{2}(k)}}{a^{2 k}}\left(\mathbf{z}-\mathbf{z}_{2}\right)^{k}+(1-\lambda) \sum_{m=1}^{N} \sum_{k=1}^{p} \frac{\alpha_{1}^{(m)}(k)}{\left(\mathbf{z}-\mathbf{z}_{1}(m)\right)^{k}}+\sum_{k=1}^{p} \frac{\alpha_{3}(k)}{\left(\mathbf{z}-\mathbf{z}_{3}\right)^{k}}\right), \\
u_{3} & =\operatorname{Re}\left(\mathbf{z}+\sum_{k=1}^{p} \frac{\overline{\alpha_{3}(k)}}{a^{2 k}}\left(\mathbf{z}-\mathbf{z}_{3}\right)^{k}+\sum_{m=1}^{N} \sum_{k=1}^{p} \frac{\alpha_{1}^{(m)}(k)}{\left(\mathbf{z}-\mathbf{z}_{1}(m)\right)^{k}}+\sum_{m=1}^{N} \sum_{k=1}^{p} \frac{\alpha_{2}^{(m)}(k)}{\left(\mathbf{z}-\mathbf{z}_{2}(m)\right)^{k}}\right),
\end{aligned}
$$

where the image points $\mathbf{z}_{1}(m)$ and $\mathbf{z}_{2}(m)$ are defined in $(15), \alpha_{j}^{(1)}(k) \equiv \alpha_{j}(k)$ for $j=1,2$, and the $\alpha_{j}^{(m)}(k)$ are defined in Lemma 2.3. Unlike the method of images, however, the coefficients $\alpha_{j}(k)$ are now unknown, and we must solve a linear system to obtain them. This is done by requiring the flux interface condition (4) to be satisfied on each inclusion boundary. In other words, the condition we impose is formally equivalent to the integral equation (7), except that the free-space kernel $G(P, Q)$ has been replaced by the more complicated $K_{j}^{\delta}(P, Q)$ :

$$
\begin{gathered}
2 \lambda_{1} n_{1}(P)=\mu_{1}(P)-2 \lambda_{1} \sum_{j=1}^{M} \int_{\partial D_{j}} \frac{\partial K_{j}^{\delta}}{\partial n}(P, Q) \mu_{j}(Q) d s, \quad P \in \partial D_{1}, \\
\ldots \\
2 \lambda_{M} n_{1}(P)=\mu_{M}(P)-2 \lambda_{M} \sum_{j=1}^{M} \int_{\partial D_{j}} \frac{\partial K_{j}^{\delta}}{\partial n}(P, Q) \mu_{j}(Q) d s, \quad P \in \partial D_{M},
\end{gathered}
$$

where $\lambda_{j}=\frac{\sigma_{j}-\sigma_{e}}{\sigma_{j}+\sigma_{e}}$, and $n_{1}(P)$ denotes the $x$-component of the unit outward normal at $P$. This leaves two questions unanswered: 1 ) in order to achieve a specified precision, how many Fourier modes $p$ are required? and 2) after how many reflections $N$ can we ignore the tail of the image series?

3.1. Error analysis. The simplest estimate for the rate of decay of the terms in an image series is obtained by examining the highest-order moment.

LEMMA 3.1. Under the conditions of Lemma $2.3, \alpha^{(m)}(p)$ satisfies the relation

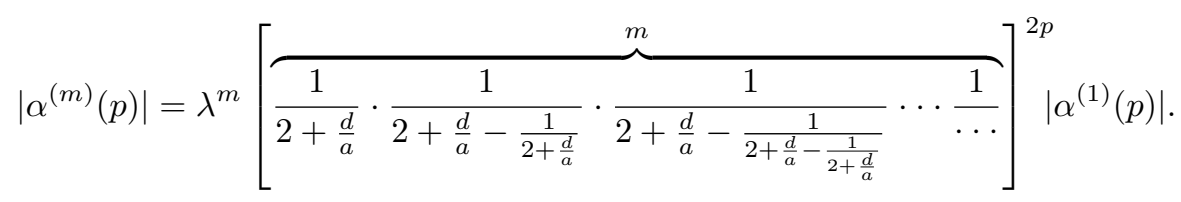

If we denote the continued fraction $f(j)$ by

$$
f(1)=\frac{1}{2+\frac{d}{a}}, \quad f(j)=\frac{1}{2+\frac{d}{a}-f(j-1)} \quad \text { for } j \geq 2,
$$


then the recursion (28) can be written as

$$
\left|\alpha^{(m+1)}(p)\right|=\lambda^{m}\left[\prod_{j=1}^{m} f(j)\right]^{2 p}\left|\alpha^{(1)}(p)\right|
$$

Proof. When $m=1$, the formula (28) is obtained immediately from (22). The general case follows by induction, observing that

$$
\overline{\alpha^{(m+1)}(p)}=-\lambda\left(\frac{a^{2}}{-\mathbf{w}_{m}^{2}}\right)^{p} \alpha^{(m)}(p),
$$

where $\mathbf{w}_{m}=\zeta_{m}-\mathbf{z}_{1}$ if $m$ is odd, and $\mathbf{w}_{m}=\zeta_{m}-\mathbf{z}_{2}$ if $m$ is even, the $\zeta_{m}$ being defined in Lemma 2.3. A tedious calculation shows that the norms of the vectors $\mathbf{w}_{m}$ satisfy

$$
\left|\mathbf{w}_{1}\right|=\left|\mathbf{z}_{1}-\mathbf{z}_{2}\right|=2 a+d, \quad\left|\mathbf{w}_{m}\right|=\left(2+\frac{d}{a}-f(m-1)\right) \cdot a \quad \text { for } m \geq 2
$$

where $f(m)$ is defined above. Therefore,

$$
\begin{aligned}
\left|\alpha^{(m+1)}(p)\right| & =\lambda\left(\frac{a}{\left|\mathbf{w}_{m}\right|}\right)^{2 p}\left|\alpha^{(m)}(p)\right| \\
& =\lambda\left(\frac{1}{2+\frac{d}{a}-f(m-1)}\right)^{2 p}\left|\alpha^{(m)}(p)\right| \\
& =\lambda(f(m))^{2 p}\left|\alpha^{(m)}(p)\right| .
\end{aligned}
$$

The worst-case scenario, of course, consists of touching disks $(d=0)$ which are superconducting $(\lambda=1)$. The continued fraction result (30) yields

$$
\left|\alpha^{(m+1)}(p)\right|=\frac{\left|\alpha^{(1)}(p)\right|}{m^{2 p}}
$$

Thus, for a 10-term multipole expansion, five reflections cause the highest-order mode to decay by a factor of approximately $10^{-14}$.

To estimate the rate of decay of lower-order multipole moments is a more subtle task. Contributions to the $k$ th reflected coefficient come from all higher-order moments of the original multipole series. It is possible to show by similar considerations that the multipole moments decay roughly at the rate

$$
\left|\alpha^{(m)}(k)\right| \approx \lambda^{m}\left[\prod_{j=1}^{m} f(j)\right]^{k}\left|\alpha^{(1)}(k)\right| .
$$

Rather than prove such a result in detail, however, we introduce a different analytic tool, based on the observation that the role of the reflection process is simply to ensure that the flux boundary condition is satisfied for the two-disk interaction.

LEMMA 3.2. Let $D_{1}$ be a disk of radius a centered at $\mathbf{z}_{1}$ and let

$$
\alpha^{(m)}(k) /\left(\mathbf{z}-\mathbf{z}_{2}(m)\right)^{k}
$$


be a reflected multipole source, where $\mathbf{z}_{2}(m)$ is defined in (15). Let $\delta=\left|\mathbf{z}_{1}-\mathbf{z}_{2}(m)\right|-$ $a>0$ and let $f_{k}^{(m)}(\mathbf{z})$ denote the contribution to the flux from this multipole source

$$
f_{k}(\mathbf{z})=\lambda \frac{\partial}{\partial n}\left(\frac{\alpha^{(m)}(k)}{\left(\mathbf{z}-\mathbf{z}_{2}(m)\right)^{k}}\right)
$$

for $\mathbf{z} \in \partial D_{1}$. Given $\epsilon>0$, if

$$
\left|\alpha^{(m)}(k)\right|<\frac{\delta^{k+1}}{k \lambda} \epsilon,
$$

then

$$
\left|f_{k}(\mathbf{z})\right|<\epsilon
$$

Proof. The maximum of $\left|f_{k}^{(m)}(\mathbf{z})\right|$ is assumed at the point $\mathbf{z} \in \partial D_{1}$ at which $\left|\mathbf{z}-\mathbf{z}_{2}(m)\right|=\delta$. A simple calculation shows that

$$
\left|f_{k}(\mathbf{z})\right|<\frac{\left|\lambda k \alpha^{(m)}(k)\right|}{\left(\left|\mathbf{z}-\mathbf{z}_{2}(m)\right|\right)^{k+1}},
$$

which yields the desired result.

Remark 3.1. The preceding lemma can clearly be generalized to the case of a full multipole expansion. However, it can also be used as a dynamic criterion in order to check, after each reflection, whether multipoles of a given order need to be retained or can be ignored. For this, let $p$ be the highest-order pole present in the $m$ th reflection. Given a tolerance $\epsilon$, all $p$ modes are retained in subsequent reflections until the criterion (32) is satisfied for $k=p$. From that point on, only terms of order $p-1$ or less are retained. This process is continued until all terms in the image multipole expansion can be ignored.

In some cases, Lemmas 3.1 and 3.2 can be used to determine a priori the number of reflections needed to resolve the close-to-touching interaction. Consider, for example, the two-disk problem described in Lemmas 2.1 and 2.3 with $a=1, d=10^{-6}$, and $\lambda=1$. Using only the dipole expansion, suppose that we wish to solve this problem to within an error of $\epsilon=10^{-6}$. The initial dipole moment is given by $\alpha^{(1)}(1)=a^{2} \lambda=1$, so that after $N$ reflections we have from Lemma 3.1

$$
\left|\alpha^{(N)}(1)\right|=\left[\prod_{j=1}^{N} f(j)\right]^{2},
$$

where $f(j)$ is defined in (29). It is easy to see that the sequence of continued fractions is increasing with limit $L$ satisfying

$$
L^{2}-(2+d) L+1=0, \quad L<1 .
$$

Since $d$ is small, a first-order solution is

$$
L \approx 1-\sqrt{d} .
$$

Thus, $\alpha^{(N)}(1)$ can be bounded by

$$
\left|\alpha^{(N)}(1)\right| \leq L^{2 N} .
$$




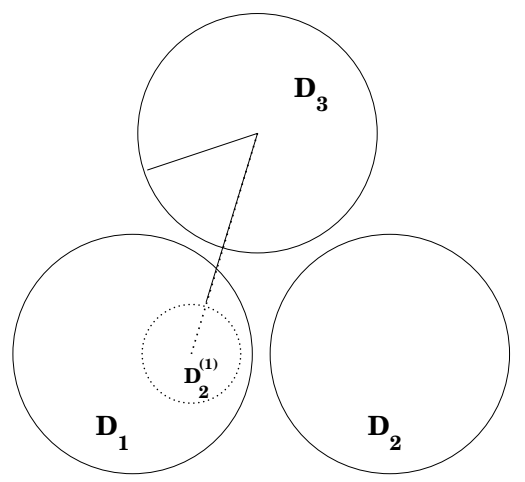

FIG. 3. Three closely spaced disks. The charge distribution $\mu_{2}$ on disk $D_{2}$ is reflected into the image disk $D_{2}^{(1)}$ inside $D_{1}$. The number of multipole moments required is determined by the condition that the field induced on $D_{3}$ by the image charge distribution on $D_{2}^{(1)}$ be well resolved (see Lemma 3.3).

According to Lemma 3.2, if we require the truncation error to be less than $\epsilon$, then we must have

$$
\left|\alpha^{(N)}(1)\right|<\delta^{2} \epsilon,
$$

where $\delta$ is the distance from the $N$ th image point, say $\mathbf{z}_{2}(N)$ in (15), to $D_{1}$. This distance satisfies

$$
\delta>\delta_{\infty}=\left|\mathbf{z}_{2}(\infty)-a\right| \geq \sqrt{d}+d / 2 \geq \sqrt{d} .
$$

The condition (35) will be satisfied if we set

$$
L^{2 N}<\delta_{\infty}^{2} \epsilon,
$$

which requires that

$$
N>\frac{\log (d \epsilon)}{2 \log L} \approx \frac{\log (d \epsilon)}{2 \log (1-\sqrt{d})} .
$$

For $d=\epsilon=10^{-6}$, the result from this estimate is 14,000 . In other words, 14,000 reflections are enough to resolve the two-disk interaction with an error less than $10^{-6}$. The actual number is less, of course, since we have been pessimistic in our use of the bounds (36) and (34).

Lemmas 3.1 and 3.2 allow us to rigorously determine the number of reflections needed to accurately compute two-disk interactions involving finite-order multipole expansions. The actual number of moments $p$ needed to properly resolve the potential field, on the other hand, is determined by multidisk interactions. Recall that $p=1$ would be sufficient if all second- and higher-order reflections were incorporated into the definition of the Green function. In our method, however, we need to increase $p$ in order to capture the effect of these higher-order reflections. The most singular interaction which we are not accounting for in our Green function is clearly that of the field induced by a first-order reflection on a third, nearby disk as depicted in Fig. 3.

LEMma 3.3. Suppose that $D_{1}, D_{2}$, and $D_{3}$ are closely spaced disks of radius a, centered at $\mathbf{z}_{1}, \mathbf{z}_{2}$, and $\mathbf{z}_{3}$, respectively. Let

$$
\Phi(\mathbf{z})=\sum_{k=0}^{p} \frac{\alpha_{2}^{(1)}(k)}{\left(\mathbf{z}-\mathbf{z}_{2}\right)^{k}}
$$


be the multipole expansion induced by a surface charge density $\mu_{2}$ on disk $D_{2}$, and let its reflection in $D_{1}$ be denoted by

$$
\Phi^{\prime}(\mathbf{z})=\sum_{k=0}^{p} \frac{\alpha_{2}^{(2)}(k)}{\left(\mathbf{z}-\mathbf{z}_{1}(2)\right)^{k}},
$$

where $\mathbf{z}_{1}(2)$ is defined in (15) and the coefficients $\alpha_{2}^{(2)}(k)$ are defined as in Lemma 2.3. Then the potential induced by this multipole expansion on the disk $D_{3}$ can be expanded as a power series

$$
\Phi(\mathbf{z})=\sum_{l=0}^{\infty} \beta_{l} \cdot\left(\mathbf{z}-\mathbf{z}_{3}\right)^{l},
$$

where

$$
\beta_{l}=\alpha_{2}^{(2)}(0) \delta_{0 l}+\sum_{k=1}^{p} \frac{\alpha_{2}^{(2)}(k)}{\left(\mathbf{z}_{1}(2)-\mathbf{z}_{3}\right)^{k+l}}\left(\begin{array}{c}
l+k-1 \\
k-1
\end{array}\right)(-1)^{k}
$$

and $\delta_{0 l}$ is the standard Kronecker delta function. Moreover, the error in truncating the infinite series (37) at $p \geq 2$ is bounded by

$$
\mathbf{E}_{p}=\left|\Phi(\mathbf{z})-\sum_{l=0}^{p} \beta_{l} \cdot\left(\mathbf{z}-\mathbf{z}_{3}\right)^{l}\right|<\frac{\|\mu\|_{L_{1}}}{c a}\left(\frac{1}{1+c}\right)^{p},
$$

where ca denotes the distance between $D_{3}$ and $D_{2}^{(1)}$ and $D_{2}^{(1)}$ is the image disk obtained upon reflection of $D_{2}$ into $D_{1}$ (see Fig. 3 and Remark 2.2).

Proof. The proof is straightforward and relies on standard multipole estimates (see [5]).

In the worst case (touching disks), we can compute from Fig. 3 that

$$
c \geq \frac{2}{3}(\sqrt{7}-2),
$$

so that the truncation error decays at least at the rate $(0.7)^{p}$.

In summary, using sufficiently many reflections, in accordance with Lemma 3.2 and sufficiently many multipole modes, in accordance with Lemma 3.3, the representation (24) yields arbitrarily accurate results.

3.2. Periodic boundary conditions. Effective transport properties of bulk materials are generally computed by considering a unit computational cell containing some number of inclusions on which one imposes periodic boundary conditions. The interface problem (1)-(4) is then augmented by the conditions

$$
\begin{aligned}
& u(x+1, y)-u(x, y)=1, \\
& u(x, y+1)-u(x, y)=0,
\end{aligned}
$$

rather than (5), to simulate a uniform field $\mathbf{E}_{a}=(1,0)$ applied in the $x$-direction [1, $2,6,15,16,33,34]$.

The standard integral equation approach, using a single layer potential, is to express $u$ as

$$
u(P)=x+\sum_{j=1}^{M} \int_{\partial D_{j}} G(P, Q) \rho_{j}(Q) d s
$$


where $G(P, Q)$ is the doubly periodic Green function rather than the fundamental solution. There are a number of questions which arise when evaluating such a Green function which we will not review in detail (see $[8,27,28]$ ). We will simply observe that periodicity can be imposed by considering the entire plane to be tiled by copies of the unit cell, generating a lattice of charge sources. Our integral equation is obtained from this potential representation, modified in the same way as the infinite medium equation, namely by incorporating all first-order reflections. That is, we solve the equation

$$
\begin{gathered}
2 \lambda_{1} n_{1}(P)=\mu_{1}(P)-2 \lambda_{1} \sum_{j=1}^{M} \int_{\partial D_{j}} \frac{\partial K_{j}^{\delta}}{\partial n}(P, Q) \mu_{j}(Q) d s, \quad P \in \partial D_{1}, \\
\ldots \\
2 \lambda_{M} n_{1}(P)=\mu_{M}(P)-2 \lambda_{M} \sum_{j=1}^{M} \int_{\partial D_{j}} \frac{\partial K_{j}^{\delta}}{\partial n}(P, Q) \mu_{j}(Q) d s, \quad P \in \partial D_{M},
\end{gathered}
$$

where $K_{j}^{\delta}$ is the doubly periodic Green function, modified to include the influence of all first-order reflections generated by interactions with disks (including periodic images) which lie within a distance $\delta$ of $D_{j}$.

4. Numerical results. Once the charge density on each inclusion boundary is expanded as a $p$-term multipole series, the integral equation (27) or (42) becomes a finite-dimensional linear system of order $M p$, as in the case of the original Fourier method described by (9). We solve this system iteratively, using the generalized minimum residual (GMRES) method [29]. At each iteration, we must perform a dense matrix-vector multiply with the actual cost determined in part by the number of reflections used in the evaluation of two-disk interactions. That number is in turn determined by the desired accuracy and Lemma 3.2.

The full algorithm has been implemented in double precision FORTRAN, including the reduction in the number of terms used in the reflection process according to Remark 3.1. Here, we illustrate its performance with a variety of examples. In all cases, the GMRES iteration was continued until the Euclidean norm of the residual was less than the desired accuracy.

Example 1. We first consider nine disks in an infinite medium (Fig. 4), each having the same conductivity but at very high contrast $\left(\sigma_{j} / \sigma_{e}=10^{8}\right)$. The closest distance between disks is denoted by $\epsilon$, and we examine the behavior of our algorithm over a range of $\epsilon$ from $10^{-2}$ to $10^{-7}$ (Table 1). For the last few cases, no previous direct data are available. In order to measure the actual error, we compare our computed solutions with those obtained using $p=50$ modes per disk and enough reflections to reduce the flux error in Lemma 3.2 to $10^{-10}$. We have also provided in Table 2 the results for the first two cases using Rayleigh's method (left group) and the algorithm of Greengard and Moura [7] (right group). While the speedup over Rayleigh's method is modest in these cases, it would be much more dramatic at smaller values of $\epsilon$. Unfortunately, Rayleigh's method is numerically unstable in that regime. The algorithm of Greengard and Moura, while numerically stable, already uses $9 \times 2000$ points for $\epsilon=10^{-3}$. It is based on a simple quadrature rule and not designed for the close-to-touching regime. Thus, we do not have competing methods available for timing comparisons in the most singular cases.

To show the nearly singular behavior of the electrostatic field at close-to-touching points, we present a contour plot of the induced potential and a surface plot of the 


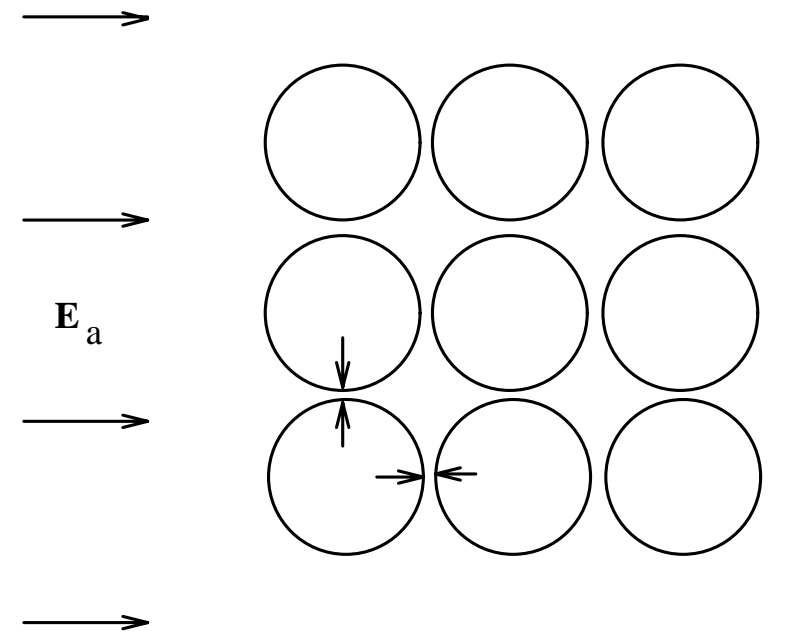

FIG. 4. Nine disks in an infinite medium, subject to a uniform applied field (Example 1). The short separation distance $\epsilon$, indicated by the arrows, is identical in the vertical and horizontal directions.

TABLE 1

Performance of the hybrid method on the nine-disk problem of Example 1. The first column indicates the separation distance $\epsilon$ and the second column indicates the number of Fourier modes $p$. The third, fourth, and fifth columns indicate the number GMRES iterations required, the computed error in the Euclidean norm, and the time required in seconds on an SGI workstation using the $\mathrm{R} 8000$ processor. The last column lists the induced net dipole moment.

\begin{tabular}{||c||c|c|c|c||c||}
\hline$\epsilon$ & Modes & \# iter & Error & Time & Total Dipole \\
\hline $10^{-2}$ & 5 & 6 & $10^{-4}$ & 0.25 & $(-0.39195,0)$ \\
\hline $10^{-3}$ & 11 & 7 & $10^{-4}$ & 1.14 & $(-0.43721,0)$ \\
\hline $10^{-4}$ & 13 & 7 & $10^{-4}$ & 3.25 & $(-0.44964,0)$ \\
\hline $10^{-5}$ & 13 & 8 & $10^{-4}$ & 10.34 & $(-0.45338,0)$ \\
\hline $10^{-6}$ & 15 & 9 & $10^{-4}$ & 38.13 & $(-0.45454,0)$ \\
\hline $10^{-7}$ & 17 & 9 & $10^{-4}$ & 53.54 & $(-0.45491,0)$ \\
\hline
\end{tabular}

TABLE 2

Performance of Rayleigh's method (columns 2-5) and the algorithm of Greengard and Moura (columns 6-9) on the nine-disk problem of Example 1. Times are again given in seconds on a SPARCstation 10. The sixth column indicates the number of points used in the discretization of each inclusion boundary (Pts).

\begin{tabular}{||c||c|c|c|c||c|c|c|c||}
\hline$\epsilon$ & Modes & \# iter & Error & Time & Pts & \# iter & Error & Time \\
\hline $10^{-2}$ & 40 & 25 & $10^{-4}$ & 9.71 & 500 & 27 & $10^{-8}$ & 90.68 \\
\hline $10^{-3}$ & 80 & 50 & $10^{-4}$ & 50.15 & 2000 & 110 & $10^{-4}$ & 1178.4 \\
\hline
\end{tabular}

absolute value of its gradient in Fig. 5. Figure 6 depicts the contours of the induced potential and a surface plot of the absolute value of its gradient for the geometry obtained by rotating the nine disk array of Figure 4 by $45^{\circ}$.

Example 2. For our second example, we consider seven irregularly placed disks in an infinite medium (Fig. 7), with conductivity ratio $10^{8}$. The closest approach distance is denoted by $\epsilon$, as in the first example. The results for this configuration 

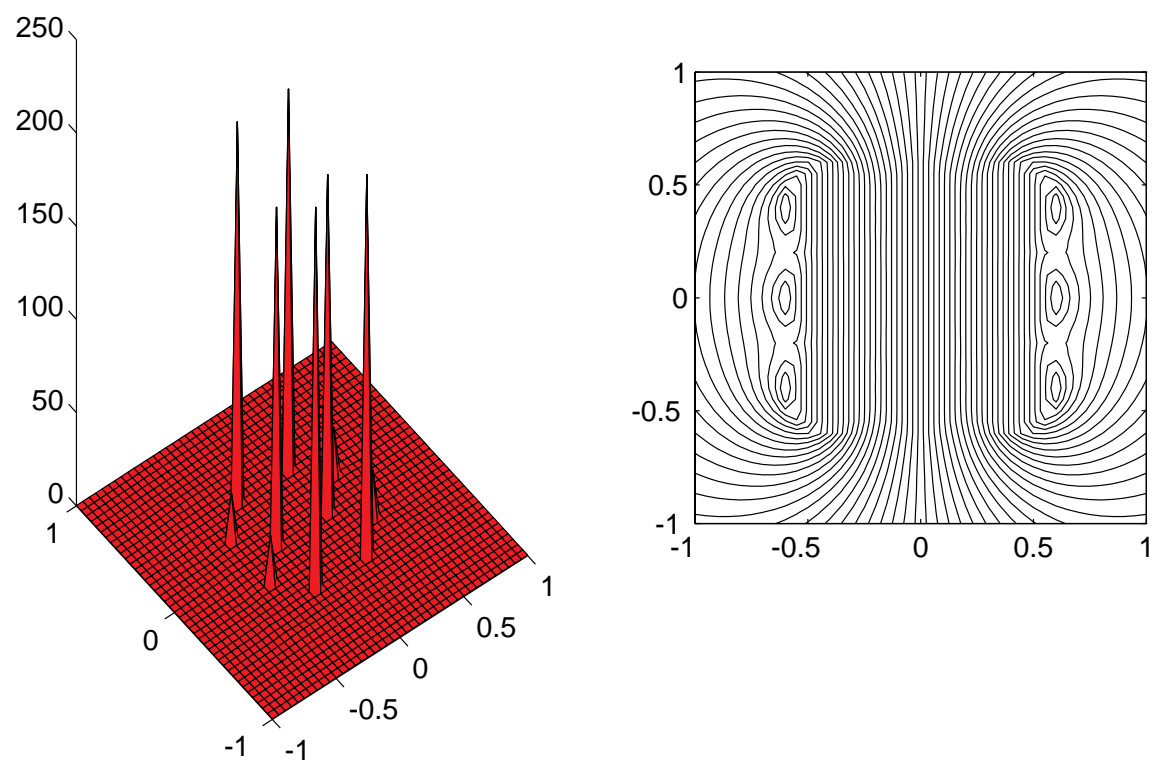

FIG. 5. A contour plot of the induced potential (right) and a surface plot of the absolute value of its gradient (left) for the case $\epsilon=10^{-5}$.
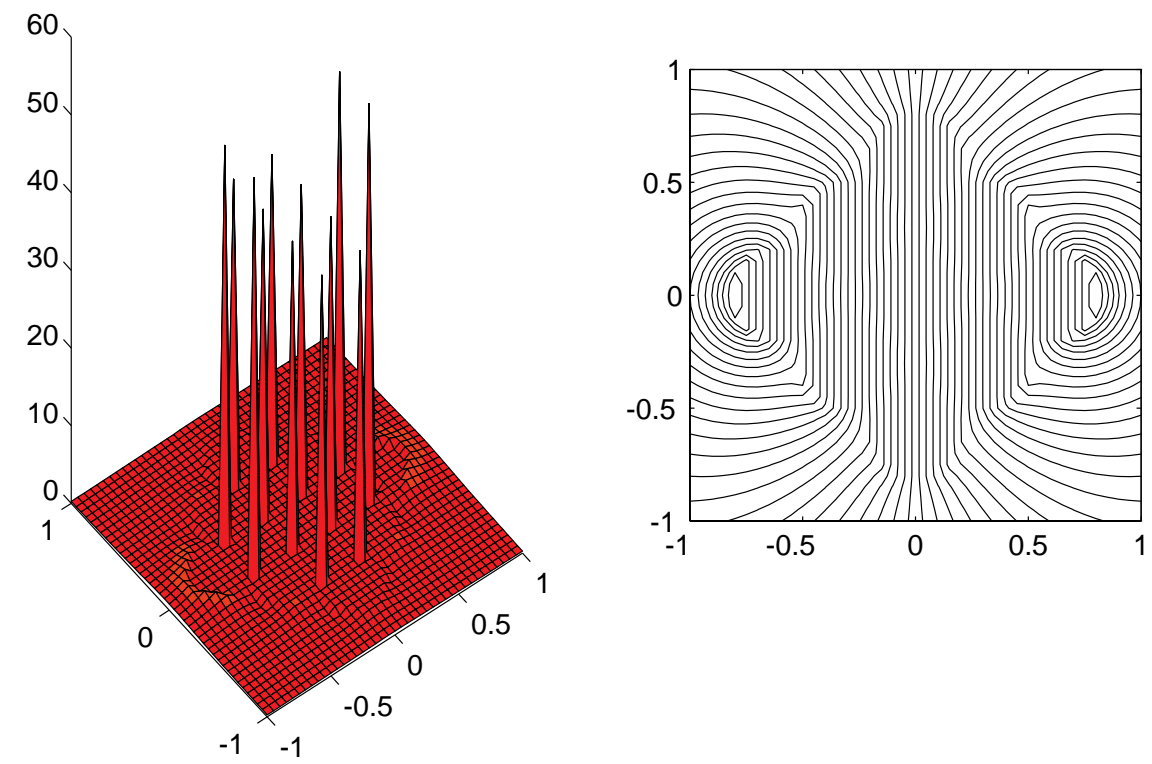

FIG. 6. A contour plot of the induced potential (right) and a surface plot of the absolute value of its gradient (left) for the geometry obtained by rotating the nine-disk array of Example 1 by $45^{\circ}$. The separation distance is $\epsilon=10^{-4}$.

are reported in Table 3. Figure 8 shows a contour plot of the potential and a surface plot of the absolute value of the potential gradient for the case $\epsilon=10^{-4}$.

Example 3. For benchmarking purposes, we have considered a (periodic) square arrays of disks. This geometry has been studied for a long time by many researchers, beginning with Rayleigh [28]. Perrins, McKenzie, and McPhedran [27] have produced 


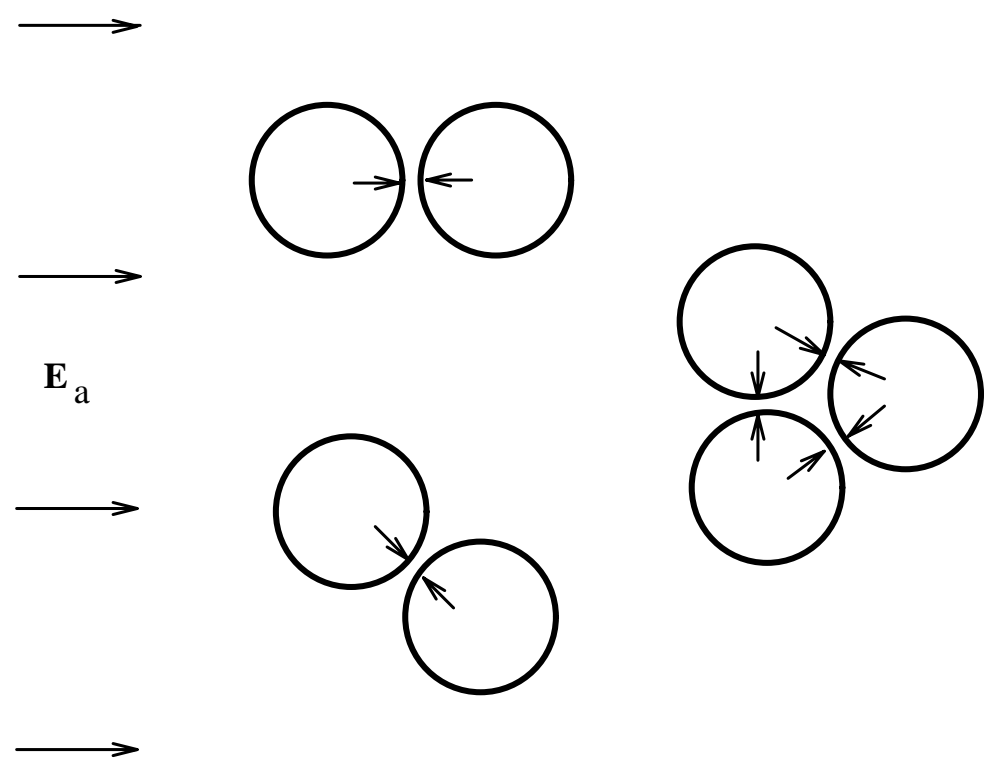

FIG. 7. The locations of seven disks in an infinite medium, subject to a uniform applied field (Example 2). The shortest separation distance $\epsilon$ is indicated by the arrows.

TABLE 3

Performance of our method on the seven-disk infinite medium problem (Example 2), with varying values of $\epsilon$. The columns are labeled as in Table 1.

\begin{tabular}{||c||c|c|c|c||c||}
\hline$\epsilon$ & Modes & \# iter & Error & Time & Total Dipole \\
\hline $10^{-2}$ & 13 & 9 & $10^{-7}$ & 0.41 & $(-0.32688,0.02337)$ \\
\hline $10^{-3}$ & 18 & 10 & $10^{-7}$ & 1.25 & $(-0.36197,0.02940)$ \\
\hline $10^{-4}$ & 19 & 11 & $10^{-7}$ & 3.49 & $(-0.37081,0.03126)$ \\
\hline $10^{-5}$ & 19 & 12 & $10^{-6}$ & 10.53 & $(-0.37336,0.03184)$ \\
\hline $10^{-6}$ & 19 & 16 & $10^{-6}$ & 40.28 & $(-0.37414,0.03202)$ \\
\hline $10^{-7}$ & 19 & 17 & $10^{-6}$ & 51.56 & $(-0.37439,0.03208)$ \\
\hline
\end{tabular}

a table which gives the effective conductivity for a wide range of area fractions and conductivity ratios. Moura [26] also considered these cases using a fast multipole accelerated integral equation. For smaller separation distances, asymptotic results have been obtained by McPhedran, Poladian, and Milton [24]. Rather than providing a complete breakdown of numerical results, we will simply observe that our results agree with previous direct calculations for modest separation distances and with the asymptotic results for the close-to-touching case. At a conductivity ratio of $10^{8}$ and a separation distance of $\epsilon=2.5 \times 10^{-4}, 13$ Fourier modes and a few seconds of CPU time on a SPARCstation 10 are required.

Example 4. In our last example, the inclusions of Example 2 are placed in a unit cell and extented periodically. Table 4 summarizes our computations.

5. Conclusions. We have presented a new integral equation method for the solution of electrostatic interface problems in systems of close-to-touching cylinders. It is based on a modified Green function which incorporates information from firstorder reflections in the method of images. Unlike previous asymptotic methods, it is 

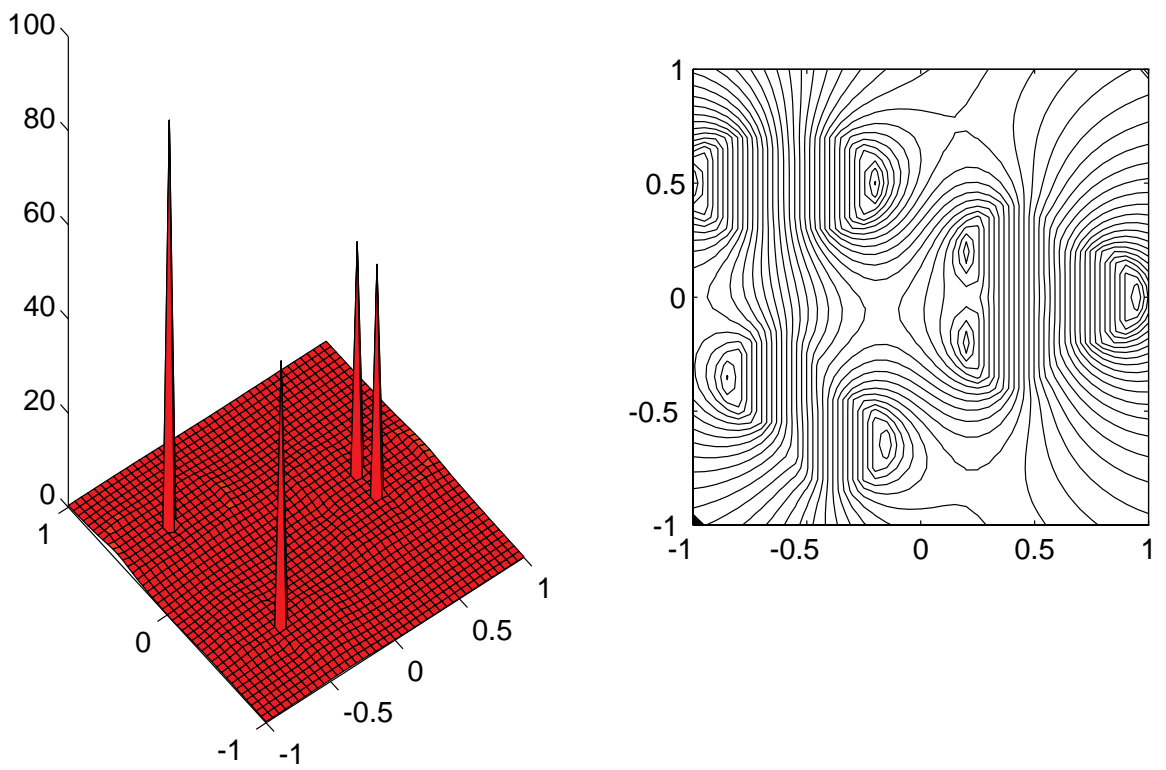

FIG. 8. A contour plot of the induced potential (right) and a surface plot of the absolute value of its gradient (left) for the case $\epsilon=10^{-4}$ in Example 2 .

TABLE 4

Performance of our method on the periodic seven-disk configuration of Example 2 with varying value of $\epsilon$. The columns are labeled as in Table 1.

\begin{tabular}{||c||c|c|c|c||c||}
\hline$\epsilon$ & Modes & \# iter & Error & Time & Total Dipole \\
\hline $10^{-2}$ & 8 & 7 & $10^{-5}$ & 0.47 & $(-0.10072,0.00548)$ \\
\hline $10^{-3}$ & 9 & 10 & $10^{-5}$ & 0.99 & $(-0.12758,0.00891)$ \\
\hline $10^{-4}$ & 9 & 11 & $10^{-5}$ & 1.88 & $(-0.13460,0.01009)$ \\
\hline $10^{-5}$ & 9 & 14 & $10^{-5}$ & 5.40 & $(-0.13661,0.01046)$ \\
\hline $10^{-6}$ & 10 & 13 & $10^{-5}$ & 14.41 & $(-0.13722,0.01058)$ \\
\hline $10^{-7}$ & 13 & 8 & $10^{-5}$ & 18.48 & $(-0.13741,0.01062)$ \\
\hline
\end{tabular}

not limited to regular geometries, and unlike previous direct numerical methods, it does not break down as the separation distance decreases toward zero.

In order to treat large numbers of inclusions, the algorithm described here, which treats neighboring interactions efficiently, should be coupled with the fast multipole method [8], which treats far field interactions efficiently. Results from such simulations will be reported at a later date.

\section{REFERENCES}

[1] G. K. BAtChelor (1974), Transport properties of two phase materials with random structure, Ann. Rev. Fluid Mech., 6, pp. 227-255.

[2] G. K. BATChelor AND R. O'BRIEn (1977), Thermal or electrical conduction through a granular material, Proc. Roy. Soc. London Ser. A., 355, pp. 313-333.

[3] C. L. Berman And L. Greengard (1994), A renormalization method for the evaluation of lattice sums, J. Math. Phys., 35, pp. 6036-6048.

[4] R. Bonnecaze And J. Brady (1990), A method to determine the effective conductivity of dispersions of particles, Proc. Roy. Soc. London Ser. A, 430, pp. 285-313. 
[5] L. Greengard (1988), The Rapid Evaluation of Potential Fields in Particle Systems, MIT Press, Cambridge, MA.

[6] L. Greengard And J. Helsing (1995), A numerical study of the $\zeta_{2}$ parameter for random suspensions of disks, J. Appl. Phys., 77, pp. 2015-2019.

[7] L. Greengard and M. Moura (1994), On the numerical evaluation of the electrostatic fields in composite materials, Acta Numerica, Cambridge University Press, Cambridge, pp. 379410.

[8] L. Greengard And V. Rokhlin (1987), A fast algorithm for particle simulations, J. Comput. Phys., 73, pp. 325-348.

[9] Z. Hashin AND S. Shtrikman (1962), A variational approach to the theory of effective magnetic permeability of multiphase materials, J. Appl. Phys., 33, pp. 3125-3131.

[10] E. Honein, T. Honein, AND G. Herrmann (1992), On two circular inclusions in harmonic problems, Quart. Appl. Math., 3, pp. 479-499.

[11] J. D. Jackson (1975), Classical Electrodynamics, Wiley, New York.

[12] M. A. Jaswon And G. T. Symm (1977), Integral Equation Methods in Potential Theory and Elastostatics, Academic Press, New York.

[13] D. J. JefFREY (1978), The temperature field or electric field around two almost touching spheres, J. Inst. Math. Applics., 22, pp. 337-351.

[14] J. Keller (1963), Conductivity of a medium containing a dense array of perfectly conducting spheres or cylinders or nonconducting cylinders, J. Appl. Phys., 34, pp. 991-993.

[15] J. Keller (1964), A theorem on the conductivity of a composite medium, J. Math. Phys., 5, pp. $548-549$.

[16] J. KeLler (1987), Effective conductivity of periodic composites composed of two very unequal conductors, J. Math. Phys., 28, pp. 2516-2520.

[17] D. Landauer (1978), Electrical conductivity in inhomogeneous media, in Electrical Transport and Optical Properties of Inhomogeneous Media, J. C. Garland and D. B. Tanner, eds. American Institute of Physics, New York, pp. 2-43.

[18] L. D. Landau, E. M. Lifshitz, And L. P. Pitaevskit (1984), Electrodynamics of Continuous Media, 2nd ed., Pergamon Press, Oxford.

[19] D. McKenzie, R. McPhedran, And G. Derrick (1978), The conductivity of lattices of spheres. II. The body centred and face centred cubic lattices, Proc. Roy. Soc. London Ser. A, 362 , pp. 211-232.

[20] R. McPhedran (1986), Transport properties of cylinder pairs and of the square array of cylinders, Proc. Roy. Soc. London Ser. A, 408, pp. 31-43.

[21] R. McPhedran, D. McKenzie, and N. Phan-Thien (1983), Transport properties of twophase composite materials, in Advances in the Mechanics and the Flow of Granular Materials, Trans Tech Publications, Clausthal, pp. 413-482.

[22] R. McPhedran And D. McKenzie (1978), The conductivity of lattices of spheres. I. The simple cubic lattice, Proc. Roy. Soc. London Ser. A, 359, pp. 45-63.

[23] R. McPhedran And G. W. Milton (1987), Transport properties of touching cylinder pairs and of the square array of touching cylinders, Proc. Roy. Soc. London Ser. A, 411, pp. 313-326.

[24] R. McPhedran, L. Poladian, and G. W. Milton (1988), Asymptotic studies of closely spaced, highly conducting cylinders, Proc. Roy. Soc. London Ser. A, 415, pp. 185-196.

[25] L. M. Milne-Thomson (1960), Theoretical Hydrodynamics, Macmillan, London.

[26] M. Moura (1993), On the Numerical Calculation of Electrostatic Fields in Composite Media, Ph.D. thesis, Courant Institute, New York University.

[27] W. T. Perrins, D. McKenzie, And R. McPhedran (1979), Transport properties of regular arrays of cylinders, Proc. Roy. Soc. London Ser. A, 369, pp. 207-225.

[28] LoRD RAYLEIGH (1892), On the influence of obstacles arranged in rectangular order upon the properties of a medium, Phil. Mag., 34, pp. 481-502.

[29] Y. SAAd AND M. H. Schultz (1986), GMRES: A generalized minimum residual algorithm for solving nonsymmetric linear systems, SIAM J. Sci. Statist. Comput., 7, pp. 856-869.

[30] A. S. SAngani And A. Acrivos (1982), The effective conductivity of a periodic array of spheres, Proc. Roy. Soc. London Ser. A, 386, pp. 263-275.

[31] A. S. Sangani And G. Mo (1994), Inclusions of lubrication forces in dynamic simulations, Phys. Fluids, 6, pp. 1653-1662.

[32] A. S. Sangani And C. YaO (1988), Transport processes in random arrays of cylinders. I. Thermal conduction, Phys. Fluids, 31, pp. 2426-2434.

[33] S. Torquato (1987), Thermal conductivity of disordered heterogeneous media from the microstrycture, Rev. Chem. Eng., 4, pp. 151-204.

[34] S. Torquato And F. LAdo (1988), Bounds on the effective conductivity of a random array of cylinders, Proc. Roy. Soc. London Ser. A, 438, pp. 591-604.

[35] J. VAn Bladel (1964), Electromagnetic Fields, McGraw-Hill, New York. 